\title{
Extension of the LOFAR Radboud Air Shower Array
}

\section{K. Mulrey ${ }^{1 *}$, A. Bonardi ${ }^{2}$, S. Buitink ${ }^{1,2}$, A. Corstanje ${ }^{1,2}$, H. Falcke ${ }^{2,3,4}$, B. M. Hare ${ }^{5}$, J. R. Hörandel ${ }^{1,2,3}$, T. Huege ${ }^{1,7}$, G. Krampah ${ }^{1}$, P. Mitra ${ }^{1}$, A. Nelles ${ }^{8,9}$, H. Pandya ${ }^{1}$, J. P. Rachen ${ }^{1,2}$, L. Rossetto ${ }^{2}$, O. Scholten ${ }^{5,6}$, S. ter Veen ${ }^{2,4}$, T. N. G. Trinh ${ }^{5,10}$, D. Veberic ${ }^{7}$, T. Winchen ${ }^{1}$}

${ }^{1}$ Astrophysical Institute, Vrije Universiteit Brussel, Pleinlaan 2, 1050 Brussels, Belgium,

2 Department of Astrophysics/IMAPP, Radboud University, P.O. Box 9010, 6500 GL Nijmegen, The Netherlands,

${ }^{3}$ NIKHEF, Science Park Amsterdam, 1098 XG Amsterdam, The Netherlands,

${ }^{4}$ Netherlands Institute of Radio Astronomy (ASTRON), Postbus 2, 7990 AA Dwingeloo, The Netherlands,

${ }^{5}$ KVI-CART, University Groningen, P.O. Box 72, 9700 AB Groningen,

${ }^{6}$ Interuniversity Institute for High-Energy, Vrije Universiteit Brussel, Pleinlaan 2, 1050

Brussels, Belgium,

${ }^{7}$ Institut für Kernphysik, Karlsruhe Institute of Technology(KIT), P.O. Box 3640, 76021,

Karlsruhe, Germany

${ }^{8}$ DESY, Platanenallee 6, 15738 Zeuthen, Germany

${ }^{9}$ ECAP, Friedrich-Alexander-University Erlangen-Nürnberg, 91058 Erlangen, Germany

${ }^{10}$ Department of Physics, School of Education, Can Tho UniversityCampus II, 3/2 Street, Ninh Kieu District, Can Tho City, Vietnam

E-mail: kmulrey@vub.ac.be

The LOFAR Radboud Air Shower Array (LORA) is an array of scintillators situated at the core of the LOFAR radio telescope. LORA detects particles from extensive air showers and acts as a trigger for the readout of the LOFAR antennas, which are densely spaced and routinely measure radio emission from air showers around $10^{17} \mathrm{eV}$. LORA originally consisted of 20 scintillators. An extension is underway that doubles the number of scintillators and increases the effective area of the array. This will result in a $45 \%$ increase in the number of triggers from higher energy cosmic rays, which are more likely to produce a strong radio signal. In addition, it will reduce the composition bias inherent in detecting low energy showers. In this contribution we discuss the status of the LORA extension and prospects for the science that can be done with the expanded triggering capabilities and improved calibration of the detector.

36th International Cosmic Ray Conference -ICRC2019-

July 24th - August 1st, 2019

Madison, WI, U.S.A.

${ }^{*}$ Speaker. 


\section{Introduction}

Cosmic rays interacting in the Earth's atmosphere create particle air showers, where radio emission is generated through the charge-excess effect and the transverse current induced by the magnetic field of the Earth. The pattern of radiation on the ground contains information about the primary cosmic ray [1], which is used to reconstruct features including energy, geometry, and composition with high precision [2]. The LOw Frequency ARray (LOFAR) is especially well suited to study radio emission from cosmic rays because of its dense antenna spacing [3, 4].

The LOfar Radboud Air shower array (LORA) consists of plastic scintillators situated at the LOFAR core. It acts as a trigger for the LOFAR antennas and provides information about the primary cosmic ray based on particle data [5]. The LORA scintillators have been recycled from the KASCADE experiment [6]. LORA provides a trigger for LOFAR antenna readout when a given number of scintillators have detected an air shower, with the trigger level for each scintillator roughly corresponding to the amount of energy deposited by one muon. In order not to interfere with regular astronomical LOFAR operations, the trigger rate must be limited to about one readout an hour.

The steeply falling cosmic ray spectrum means that most triggered events are low in energy, around $10^{16} \mathrm{eV}$, where the radio signal is unlikely to be detectable above background noise. There is also a composition bias introduced by the triggering scheme that has to be taken into account, due to the fact that air showers from lighter primaries are more likely to produce a trigger. LORA originally consisted of 20 scintillators located on the superterp, the most densely populated region of antennas in the LOFAR core. In order to increase the number of quality events detected, an expansion of LORA is underway which will add 20 new scintillators to the array. It will reduce the trigger bias and increase the effective area. We have also revisited the calibration of the new and existing LORA scintillators in order to reduce the systematic uncertainties in the measurements and better understand biases in triggering on particles.

\section{Expansion}

Currently, only $19 \%$ of LORA triggered events have a detectable cosmic-ray radio signal. This is because LORA triggers primarily on showers that are below $10^{16.5} \mathrm{eV}$. Triggering on only high energy showers could be achieved by increasing the number of detectors necessary to form a trigger. However, this reduces the trigger rate to an unnecessarily low level. Furthermore, many events that do contain a radio signal suffer from triggering biases introduced by triggering on particles. Since a primary science result for LOFAR cosmic rays is composition studies, decreasing the composition bias will increase the number of usable events.

In order to design the extension of LORA, a simulation study was carried out to determine the optimal layout for the 20 new scintillators at the LOFAR core using CORSIKA 7.4387 [7], with QGSJET II-04 [8], FLUKA [9], and GEANT4 [10]. Details of the simulations can be found in [11]. Consideration had to be taken to install the new scintillators at existing LOFAR antenna stations where the infrastructure was already in place. Scintillators were grouped in sets of four. The resulting layout is shown in the left panel of Figure 1. The blue squares indicate the positions of the existing scintillators, and the red squares indicate the positions of the new ones. The right 
panel of Figure 1 shows the positioning of the scintillators relative to the antenna fields (note: scintillators are roughly $1 \mathrm{~m} \times 1 \mathrm{~m}$ and are not shown to scale).
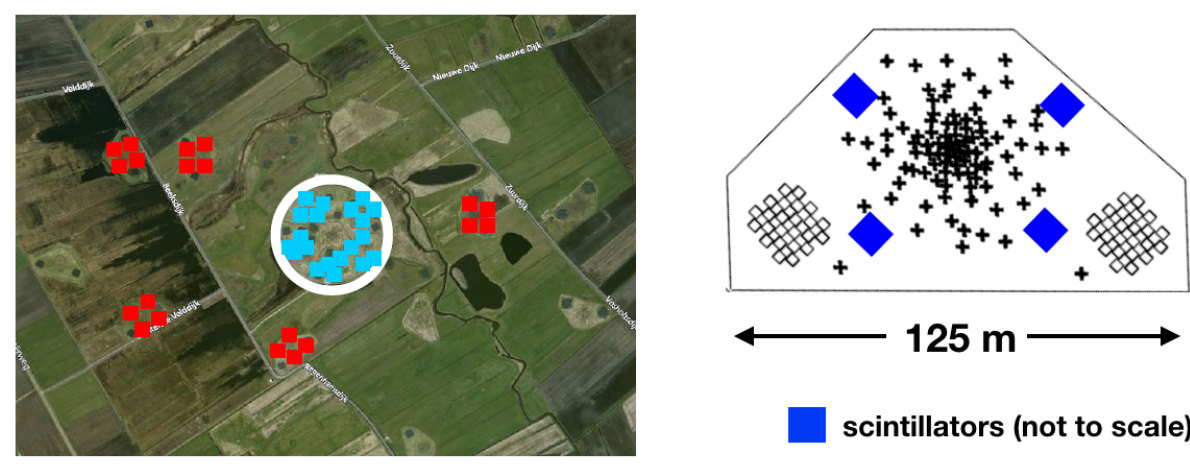

scintillators (not to scale)

\section{$2 \mathrm{~km}$}

Figure 1: Layout of the new LORA stations. Left: New scintillator positions are shown in red, and existing ones in blue. The white circle indicates the position of the superterp, which has the densest antenna spacing. Right: Positioning of the scintillators relative to the antenna fields. Scintillators are not shown to scale. Crosses indicate low band $(30-80 \mathrm{MHz})$ antennas, and boxes indicate high band $(120-240 \mathrm{MHz})$.

With the added LORA stations, the trigger requirement will be adjusted so that the rate remains at 1 trigger per hour. This means the minimum number of panels required to form a trigger increases, thereby limiting the number of low energy showers triggering. The increased effective area also allows for triggering on higher energy showers. The results of the increased effective area as determined by simulation are shown in the left panel of Figure 2, where a $45 \%$ increase in usable events is expected.

A second benefit of adding more scintillators to the array is that the effect of triggering bias is reduced. Because heavier composition cosmic rays interact further up in the atmosphere, the shower develops higher up, and more particles are attenuated by the time the shower front reaches ground level. This means that showers initiated by lighter particles have a higher chance of triggering. This effect is enhanced at higher zenith angles. In order to eliminate trigger biases, we check on an event-to-event basis that the geometry of the event is such that both proton and iron initiated showers would generate a trigger. This results in most events below $10^{17} \mathrm{eV}$ being discarded. By adding more scintillators, we increase the probability that more showers trigger. This is shown in the right panel of Figure 2. The probability of detecting proton and iron showers with a core within $250 \mathrm{~m}$ of the center of the superterp is shown as a function of the number of scintillators required for a trigger. With the new extension, both primaries trigger at close to $100 \%$ down to $10^{16.5} \mathrm{eV}$ for a trigger condition of 13/20 scintillators with signal. This increases the number of quality events, in addition to the $45 \%$ increase due to the expanded effective area.

The left panel of Figure 3 shows the locations of shower cores triggered with the original LORA configuration in red, and with the expansion in blue, with trigger conditions such that the trigger rate remains at 1 event per hour (13/20 scintillators for the original array and 14/20 for the extended array). As well as increasing the effective area for triggering, triggering with cores well 

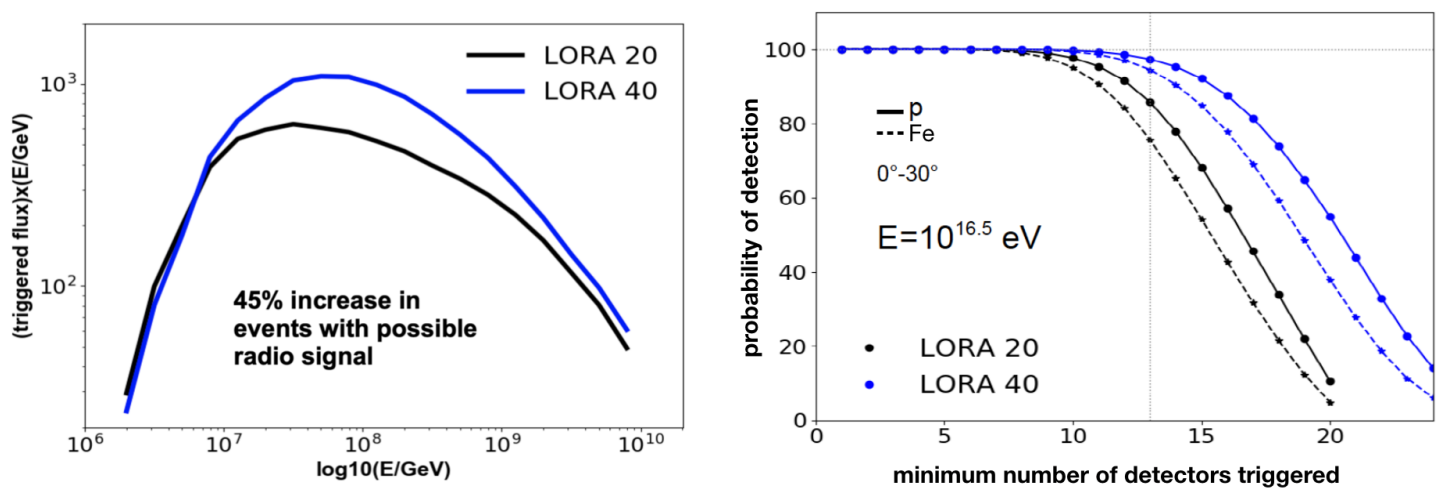

Figure 2: Left: CR flux multiplied by the probability of triggering. The trigger condition is chosen to keep the rate under 1 event per hour, and so different detector arrangements have different conditions. Right: Probability of triggering a minimum number of detectors for a shower of energy $10^{16.5} \mathrm{eV}$ within $250 \mathrm{~m}$ of the center of the superterp. Solid lines represent proton showers and dashed represent iron showers.

outside the superterp will allow the densest antenna area to probe the shower footprint outside the Cherenkov cone, where the emission from all along the shower track is less compressed in time, providing more information about shower development. An example radio footprint for a $10^{17} \mathrm{eV}$, $45^{\circ}$ air shower in relation to LOFAR low-band antennas is shown in the right panel of Figure 3.
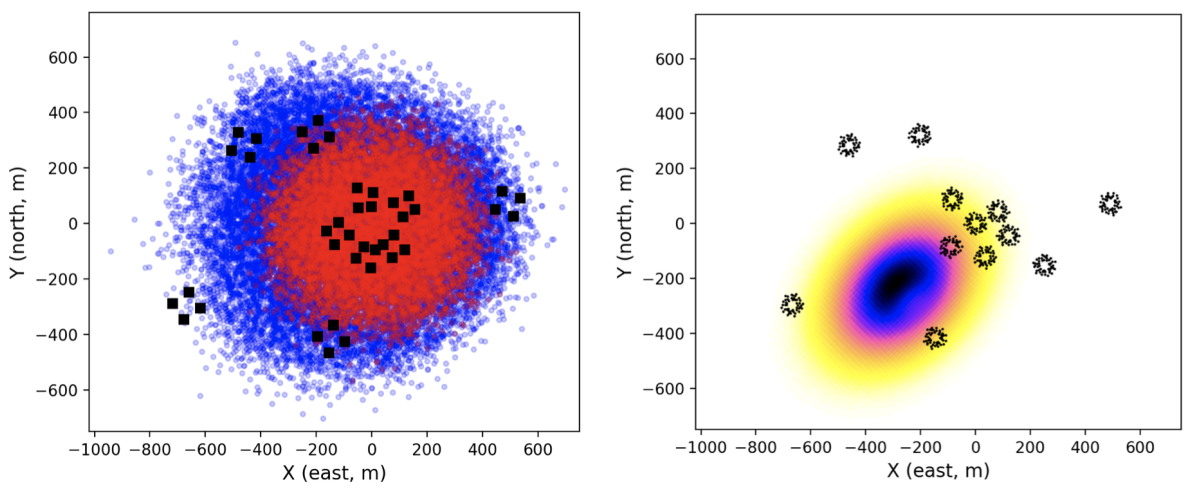

Figure 3: Left: Positions of cores of showers triggered with the original 20 scintillators (red) and the new arrangement (blue) for showers with energy $10^{17} \mathrm{eV}$. The black squares indicate the positions of scintillators. Right: Sample simulated radio footprint for a shower of $10^{17} \mathrm{eV}$ and $45^{\circ}$ zenith, with positions of the LOFAR antennas.

The first stage of the extension took place in the spring of 2018, when the infrastructure for the new scintillators was installed. This included laying cabling and adapting the central LOFAR electronics cabinets. The scintillators were calibrated in the laboratory and installed in the field in spring of 2019. Data taking is expected to commence in early fall of 2019.

\section{Improved calibration}

During the LORA expansion we have revisited the calibration of the scintillators in order to reduce systematic uncertainties and better understand biases in the trigger system. We studied the 
calibration based on the energy deposited by single charged particles, the spatial dependence of the scintillator response, and reflections in the cabling.

\subsection{Deposited energy from single muons}

It is important to understand the integrated ADC counts of a trace produced by a single muon, which is a proxy for energy deposited in the scintillator. Here we compare three sets of data: simulated muon energy deposits, muons measured with a LORA scintillator in a muon tracking detector, and muons measured with a LORA scintillator using the electronics that will be used in the LORA extension.

The conversion from integrated ADC counts to deposited energy is done by simulating the detector response to all-sky muons and comparing the expected energy deposit to the measured deposit. Geant 4 simulations are used, and more details of the simulation procedure can be found in [5]. The distribution of simulated energy deposits is shown as the orange curve in the right panel of Figure 4. The most probable energy deposit in the scintillator is $6.3 \mathrm{MeV}$, but the distribution here is scaled so that the most probable value (MPV) is 100 integrated counts to be comparable with the other measurements. The shape follows the expected Landau distribution.

Four scintillators were sent to Karlsruhe Institute of Technology (KIT) to be measured in a muon tracking detector which was originally used for muon tracking in the KASCADE experiment [6]. This measurement allowed us to study the distribution of deposited muon energy as a function of position in the scintillator and with high statistics. The all-sky muon energy deposits are shown as the blue curve in the right panel of Figure 4, scaled to MPV=100 counts. The shape of the distribution matches well with expectation from simulations.

Additionally, we calibrated all the new scintillators by measuring the energy deposits (i.e. integrated ADC counts) of single muons with the electronics that will be used in the field. In order to make use of the full dynamic range of the digitizing electronics, we set the gain so that the signal from a single muon is close to the noise level. This way we avoid saturation when measuring strong signals from air showers. A sample muon trace is shown in the left panel of Figure 4. The red overlay shows the integration window, which extends from -70 to $+300 \mathrm{~ns}$ from the position of the pulse peak. The resulting distribution is shown in green in the right panel of Figure 4, again scaled to MPV=100. The distribution of deposits from single muons measured close to the noise level is not as clean as the distribution from simulations from the KIT measurements, as the background noise can add constructively or destructively. However, the shapes of all three distributions agree for deposits above the MPV. The noise floor is also clearly separable from the muon peak. With this knowledge, we can determine the correct conversion from measured ADC counts to deposited energy.

\subsection{Spatial dependence of the scintillator response}

In order to find the particle-based energy for a cosmic-ray event, particles from CORSIKA air showers reaching ground level are run through a Geant4 simulation using a realistic model of the scintillators [10]. For this reason, it is important to know the spatial dependence of the scintillator responses. This was measured in the KIT muon tower for 4 scintillators. The layout of a scintillator is shown in the left panel of Figure 5. Each unit contains two PMTs which see 

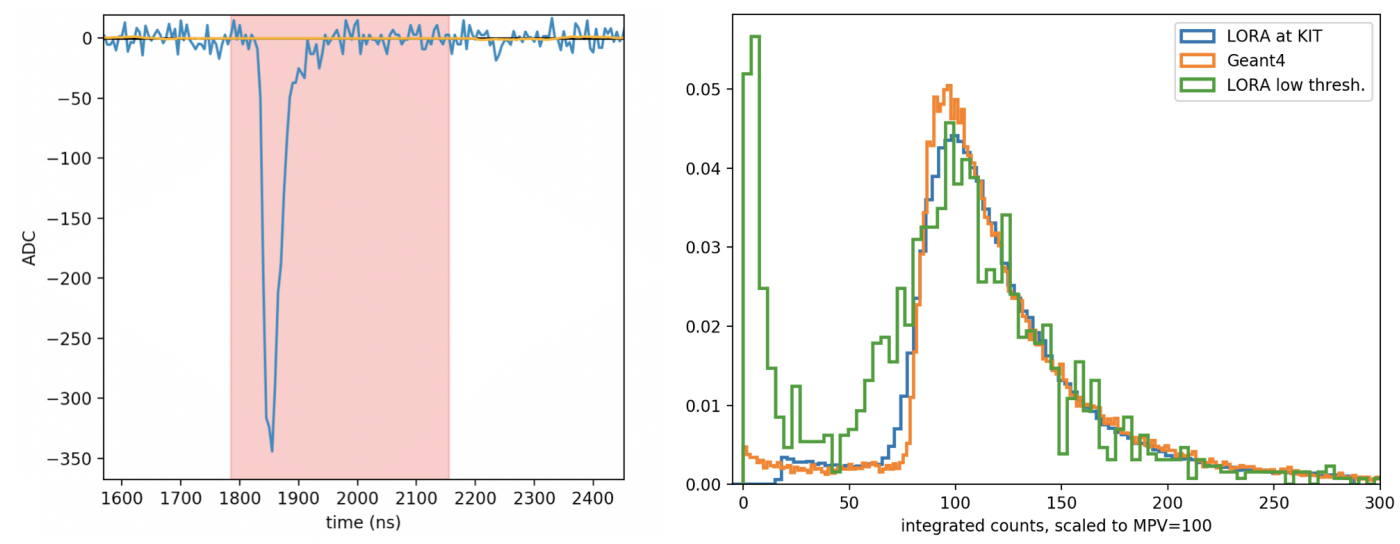

Figure 4: Left: Example trace of a single muon in a LORA scintillator. The red overlay indicates the integration window for determining the total ADC counts, which corresponds to energy deposited in the scintillator. Right: Distributions of energy deposits by singly charged particles in the scintillator, scaled so that the most probable value is 100 . The blue line indicates measurements made in the KIT muon tower, orange indicates Geant 4 simulated muons, and green indicates muons measured close to the noise floor.

light from two scintillating panels each, connected with a wavelength shifting bar. The right panel shows the deposited charge as a function of position along the $\mathrm{x}$-axis of the scintillator, as defined in the left panel, for four PMTs. Besides a deposit offset between some pairs, the distribution of charge along the panel is relatively constant, differing most noticeably with an increase near the wavelength shifter. This spatial dependence can now be included in simulations.

\subsection{Cable reflections}

The signal carrying coaxial cables from two PMTs are joined using a BNC splitter. This causes a drop in signal from each PMT, as well as a reflection of the main signal. An example is shown in Figure 6. In the left panel, a signal is shown directly from the PMT (top), and after a BNC splitter is used to join the signal from two PMTs (bottom). Calibration data is taken for each scintillator both with and without reflections.

The right panel shows a scatter plot of integrated ADC counts (a proxy for deposited charge), against the peak ADC count in the trace, for data collected with reflections (in green) and without reflections (in blue). Histograms of the distributions projected along each axis are shown on the side panels. Here, it can be seen that the integrated counts remain similar in both cases, which means the energy conversion from $\mathrm{ADC}$ counts to $\mathrm{MeV}$ is unaffected. This is because the reflected pulse is also within the integration window. We find that the integrated signal drops between 0 and $10 \%$ across all scintillators when reflections are included. This is added to the systematic uncertainties on the energy scale derived from particle data. The peak ADC value drops by closer to $40 \%$ however, which must be taken into consideration when handling trigger biases, as the trigger is based on signal over threshold.

\section{Conclusion}

LORA acts as a trigger for radio readout for cosmic-ray detection at the LOFAR telescope, and 

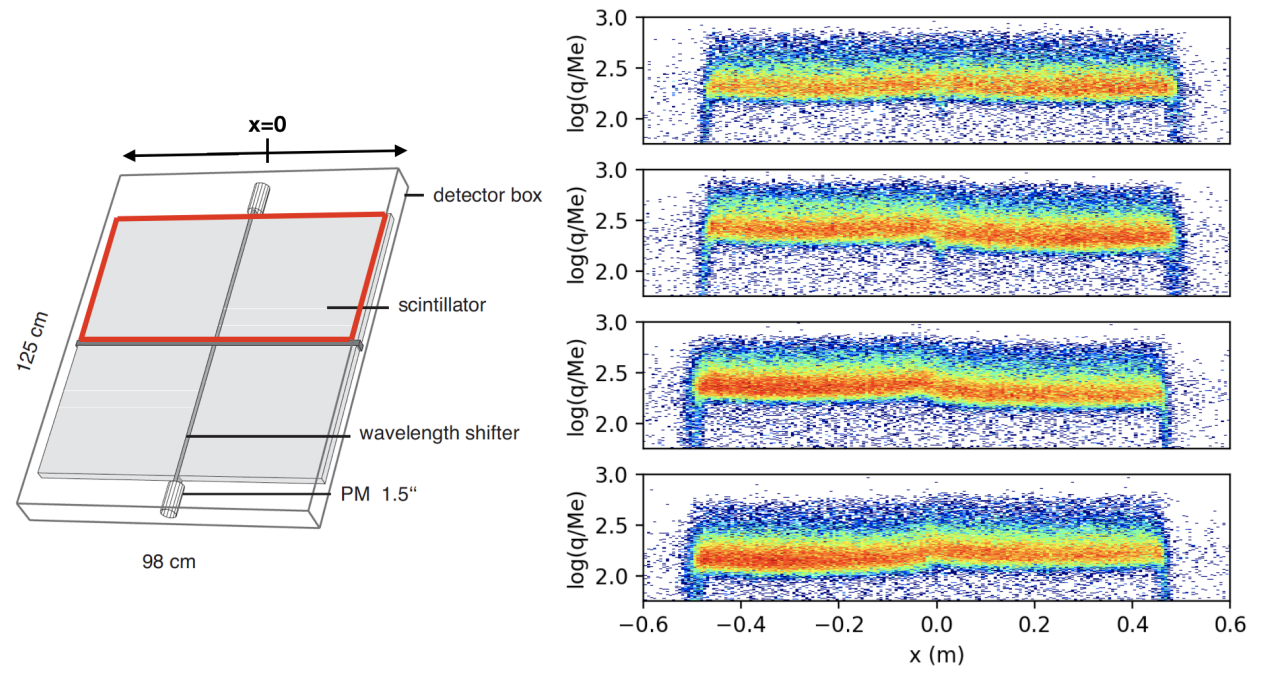

Figure 5: Left: Schematic view of the LORA scintillators. One PMT sees light from two panels joined by a wavelength shifter, indicated by the red box. Original scintillator schematic adapted from [6]. Right: Deposited charge as a function of position along the $\mathrm{x}$-axis of the scintillator, as defined in the left panel. The four plots are examples of different panel pairs. The color scale indicates the counts in each bin.

provides particle-based information about the primary cosmic ray used in event reconstruction. An expansion is underway that doubles the number of scintillators and expands the effective area of the array. This will increase the number of measured cosmic-ray events with a strong radio signal by $45 \%$, and also decrease the trigger bias against heavier elements prominent in low-energy events. A study of the response of the scintillators has also been carried out. Measurements at the KIT muon tower provided information about the spatial response of the detectors, which will be taken into consideration in event simulation and reconstruction. System responses such as reflections in the cables can also be treated in data processing, to ensure that trigger biases are handled correctly. The LORA expansion is well underway, with field deployment of the scintillators having taken place in the spring of 2019. First data is expected in the coming months.

\section{Acknowledgements}

The LOFAR cosmic-ray key science project acknowledges funding from an Advanced Grant of the European Research Council (FP/2007-2013) / ERC Grant Agreement n. 227610. The project has also received funding from the European Research Council (ERC) under the European Union's Horizon 2020 research and innovation programme (grant agreement No 640130). We furthermore acknowledge financial support from FOM, (FOM-project 12PR304). TW is supported by DFG grant WI 4946/1-1. AN is supported by the DFG grant NE 2031/2-1. LOFAR, the Low Frequency Array designed and constructed by ASTRON, has facilities in several countries, that are owned by various parties (each with their own funding sources), and that are collectively operated by the International LOFAR Telescope foundation under a joint scientific policy. 

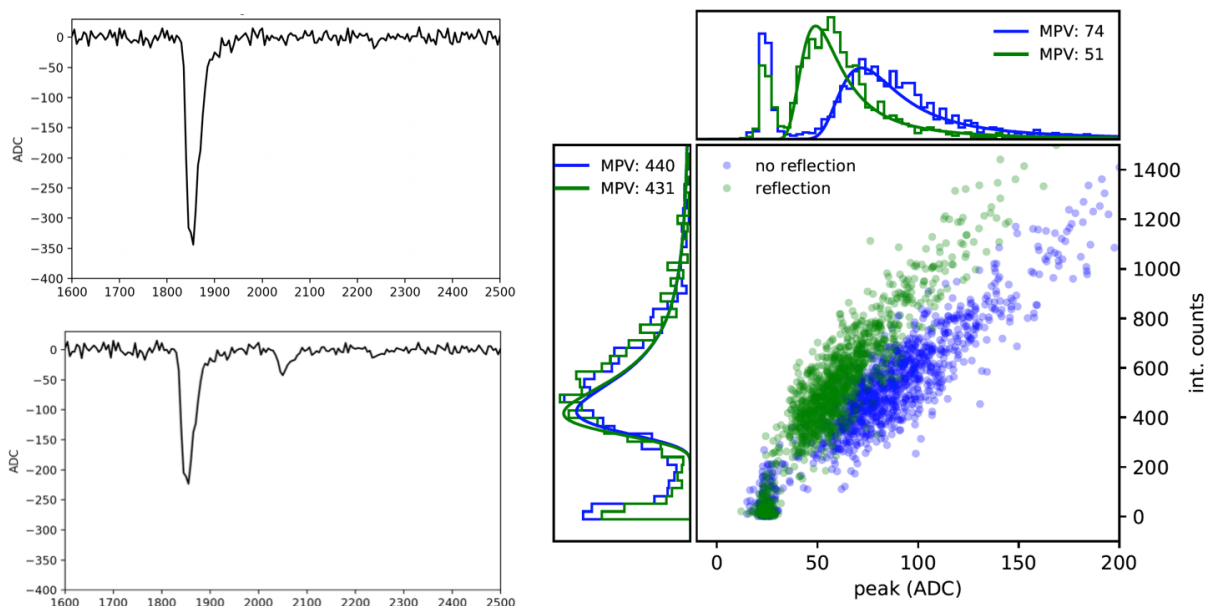

Figure 6: Left: sample traces from one PMT. Top: Single muon trace directly from one PMT. Bottom: Trace from a single muon, with signals from two PMTs joined with a BNC splitter. Right: Scatter plot of integrated ADC counts (a proxy for deposited charge), against the peak ADC count in the trace, for data collected with reflections (in green) and without reflections (in blue). Histograms of the distributions projected along each axis are shown on the side panels.

\section{References}

[1] T. Huege. Radio detection of cosmic ray air showers in the digital era. Physics Reports, 620:1-52, 2016.

[2] S. Buitink et al. Method for high precision reconstruction of air shower $\mathrm{X}_{\max }$ using two-dimensional radio intensity profiles. Phys. Rev. D, 90(8), 2014.

[3] P. Schellart et al. Detecting cosmic rays with the LOFAR radio telescope. Astronomy and Astrophysics, 560(A98), 2013.

[4] M. P. van Haarlem et al. LOFAR: The LOw-Frequency ARray. Astronomy and Astrophysics, 556:56, 2013.

[5] S. Thoudam et al. LORA: A scintillator array for LOFAR to measure extensive air showers. Nucl.Instrum.Meth, A767:339-346, 2014.

[6] T. Antoni et al. (KASCADE Collaboration). The cosmic-ray experiment KASCADE. Nuclear Instruments and Methods A, 513:490, 2003.

[7] D. Heck et al. CORSIKA: A Monte Carlo code to simulate extensive air showers. Report FZKA, 6019, 1998.

[8] S. Ostapchenko. Monte Carlo treatment of hadronic interactions in enhanced Pomeron scheme: QGSJET-II model. Phys. Rev. D, 83(014018), 2011.

[9] A. Ferrari et al. FLUKA: a multi-particle transport code. CERN-2005-10, INFN/TC_05/11, 2005.

[10] S. Agostinelli et al. GEANT4 -a simulation toolkit. NIMPA, 506(3):250-303, 2003.

[11] K. Mulrey et al. Expansion of the LOFAR Radboud Air Shower Array. PoS, ICRC2017:413, 2018. 